\title{
Estudio mecánico de un acero cromo-molibdeno con recubrimiento duro
}

\author{
Mechanical Study of a Chrome-Molybdenum Steel with Hard Coating \\ Daniel Sánchez-Huerta ${ }^{1}$, José Antonio Juanico-Loran ${ }^{3}$ \\ Víctor Jorge Cortes-Suarez ${ }^{2}$, Noé López-Perrusquia ${ }^{4}$ \\ 1 Universidad Autónoma Metropolitana | Universidad Politécnica del Valle de México, MÉXIco \\ https://orcid.org/0000-0002-4762-2160 | ingenierodaniel@outlook.com \\ 2Universidad Autónoma Metropolitana, MÉXICO \\ vjcs@azc.uam.mx \\ 3Universidad Politécnica del Valle de México, MÉxIco \\ https://orcid.org/0000-0003-4423-6552 | jjuanico@upvm.edu.mx \\ 4Universidad Politécnica del Valle de México, MÉXICo \\ https://orcid.org/0000-0001-9209-8612 | nlopezp@upvm.edu.mx
}

\section{Resumen}

Se evalúa el tratamiento termoquímico de boro a temperaturas de 950 y $1000{ }^{\circ} \mathrm{C}$, con un tiempo de exposición de 2 y 0.5 h; la adhesión de la capa se analiza mediante la técnica de Rockwell C prescrita por la norma alemana VDI 3198 y obtener una clasificación cualitativa; por microscopía óptica se observa la tasa de crecimiento y la morfología de la capa de $\mathrm{FeB} / \mathrm{Fe}_{2} \mathrm{~B}$ en la superficie del material; la presencia de fases $\mathrm{FeB} / \mathrm{Fe}_{2} \mathrm{~B}$ se determinó por difracción de rayos X (XRD). La técnica de microscopía electrónica de barrido (EDS) muestra la distribución de los elementos de aleación en la capa del sustrato $\mathrm{FeB} / \mathrm{Fe}_{2} \mathrm{~B}$ formada en la superficie del acero de medio carbón. El módulo elástico y la dureza se obtuvieron con ensayos de nanoindentación en la capa bifásica generada. Esta investigación tiene como objetivo caracterizar la morfología, comportamiento adhesivo y mecánico del recubrimiento de boruro formado en un acero AISI 4140 mediante la técnica de pasta deshidratada, con bajos tiempos de difusión que evitan la formación en demasía de la fase frágil FeB.

Palabras clave: adherencia, borurado, delaminación.

\begin{abstract}
The thermochemical treatment of boron is evaluated at temperatures of 950 and $1000^{\circ} \mathrm{C}$, with an exposure time of 2 and $0.5 \mathrm{~h}$; the adhesion of the layer is analyzed using the Rockwell C technique by the German VDI 3198 standard and a qualitative classification is obtained. By optical microscopy the growth and morphology of the $\mathrm{FeB} / \mathrm{Fe}_{2} \mathrm{~B}$ layer on the surface of the material is observed; the presence of $\mathrm{FeB} / \mathrm{Fe}{ }_{2} \mathrm{~B}$ phases was determined by $\mathrm{X}$-ray diffraction (XRD). The scanning electron microscopy (SEM) technique shows the distribution of the alloy elements in the substrate $\mathrm{FeB} / \mathrm{Fe}_{2} \mathrm{~B}$ layer formed on the surface of the medium carbon steel. The elastic modulus and hardness obtained with nanoindentation tests on the generated two-phase layer. The aim of this research is to characterize the morphology, adhesive and mechanical behavior of the boride coating formed on an AISI 4140 steel by means of the dehydrated paste technique, with low diffusion times that prevent the formation of too much of the fragile FeB phase.
\end{abstract}

Index terms: adherence, boriding, delamination. 


\section{INTRODUCCIÓN}

En la manufactura industrial se utilizan diferentes tratamientos térmicos y recubrimientos que generan características mecánicas beneficiosas en un material metálico, el recubrimiento de boruro, genera aumento en la dureza superficial del material. Cuando un material metálico ferroso de medio carbón y baja aleación, es sometido a un tratamiento termoquímico de boro, adquieren altas durezas superficiales (1600 a $2000 \mathrm{Mpa}$ ) debido a la difusión de átomos de boro en el acero, generando capas tipo $\mathrm{Fe}_{2} \mathrm{~B}$ y $\mathrm{FeB}$; estas fases se forman por la calidad en el proceso de borurado y por los elementos químicos que se encuentran en el acero a tratar termoquímicamente, siendo eficiente en aceros con bajos aleantes o microaleantes [1], [2].

Los recubrimientos duros se han estudiado para aplicaciones que beneficien el entorno industrial, por mencionar algunas: corrosión, fricción, cargas estáticas, cargas dinámicas, entre otros; generadas por las necesidades del proceso de producción de las empresas. Para tener un comportamiento adecuado, se estudia la adherencia del recubrimiento duro de boro. Existen diferentes pruebas que miden la calidad de los recubrimientos duros, la que se utilizara es mediante cargas mecánicas [3], [4] utilizadas para determinar la calidad de la adherencia en un recubrimiento duro, se realiza con una indentación Rockwell C prescrita en la norma VDI 3198 (véase Fig. 1). El principio de este método consta de un penetrador de geometría circular, penetra en la superficie de un material cubierto, lo que induce la deformación plástica masiva al sustrato y la fractura del recubrimiento.

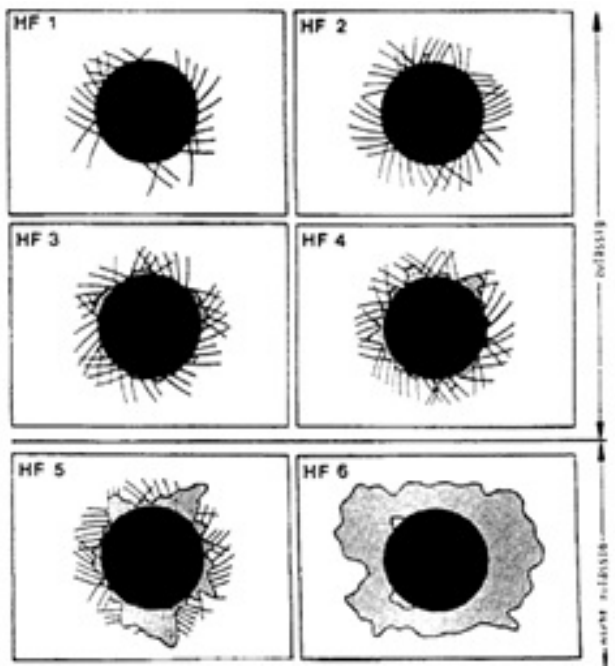

Fig. 1. Evaluación de la adherencia de la capa en el substrato mediante la norma VDI 3198 [7]

El objetivo del presente estudio es determinar cualitativamente la adhesión, por la prueba de Rockwell-C. Se evalúa la adherencia de un recubrimiento bifásico en un acero de medio carbón con capa de boruro de hierro producido por un tratamiento termoquímico de pasta deshidratada. Se realiza este tipo de pruebas por la simplicidad con la que se puede llevar a cabo, tanto en el área de investigación como en cualquier industria de manufactura, obteniendo la información básica de las características mecánica y así poder generar diseños de elementos mecánicos con recubrimientos de boro que se acoplen a las necesidades de los procesos industriales [5], [6].

\section{DesarRollo eXPERIMENTAL}

\section{A. Proceso de borurado}

El tratamiento termoquímico de borurización se llevó a cabo a muestras de acero AISI 4140, con un diámetro de $25.4 \mathrm{~mm}$. Posteriormente el proceso de borurado se realiza por la técnica de caja, con pasta de boro deshidrata en 
polvo $\left(\mathrm{B}_{4} \mathrm{C}+\mathrm{Na}_{3} \mathrm{AlF}_{6}\right)$. Las temperaturas de tratamiento fueron 950 y $1000^{\circ} \mathrm{C}$; con permanencia de $0.5 \mathrm{y} 2 \mathrm{~h}$, para cada espécimen a la temperatura indicada. Las muestras boruradas se prepararon para metalográfica, se mide el espesor de la capa de boruro de hierro en un microscopio óptico, Olympus GX; las mediciones de las capas se estimaron con el software imagen pro-plus $\mathrm{v}$ 4.0. Se determina el módulo de elasticidad por la técnica de nanoindentación.

\section{B. Adherencia}

El estudio de adherencia se realizó por el ensayo de Rockwell-C con un equipo Mitutoyo; determinando cualitativamente la adhesión de la capa formada en la superficie del acero AISI 4140 con tratamiento termoquímico de borurado. Los resultados de la capa fracturada se compararon con la norma de adherencia VDI 3198.

\section{Nanoindentación}

Se realiza la nanoindentación a cuatro pruebas con temperatura de 950 y $1000^{\circ} \mathrm{C}$ y tiempo de exposición de 0.5 y $2 \mathrm{~h}$, se efectúan ensayos de indentación, desde la superficie hasta el material base, obteniendo las gráficas esfuerzo-desplazamiento, la nanoindentación se centra en las fases $\mathrm{Fe}_{2} \mathrm{~B}$ y FeB generadas por el tratamiento de borurado.

\section{Microscopio óptico}

Se estudia mediante microscopia óptica la formación de las fases $\mathrm{Fe}_{2} \mathrm{~B}$ y FeB en el recubrimiento de las muestras tratadas termoquímicamente a 950 y $1000^{\circ} \mathrm{C}$, se estudia el espesor bifásico $\mathrm{Fe}_{2} \mathrm{~B}$ y $\mathrm{FeB}$ generado en cada muestra.

\section{RESULTADOS Y DISCUSIONES}

Se tiene un espesor de crecimiento en función de las temperaturas y tiempos de permanencia de estudio, como se muestra en la Tabla I.

TABLA I

ESPESORES DEL RECUBRIMIENTO BIFÁSICO DE BORO EN EL ACERO AISI 4140

\begin{tabular}{ccrr}
\hline \multirow{2}{*}{$\begin{array}{c}\text { Temperatura } \\
\left({ }^{\circ} \mathbf{C}\right)\end{array}$} & $\begin{array}{c}\text { Tiempo de } \\
\text { permanencia }(\mathbf{h})\end{array}$ & \multicolumn{2}{c}{$\begin{array}{c}\text { Espesor promedio del boruro } \\
(\boldsymbol{\mu m})\end{array}$} \\
\cline { 3 - 4 } & 0.5 & \multicolumn{1}{|c|}{$\mathbf{F e}_{2} \mathbf{B}$} & \multicolumn{1}{c}{$\mathbf{F e B}$} \\
\hline \multirow{2}{*}{950} & 2.0 & $28.42 \pm 9.29$ & $4.65 \pm 1.99$ \\
& 0.5 & $46.70 \pm 13.10$ & $17.82 \pm 6.88$ \\
\multirow{2}{*}{1000} & 2.0 & $30.31 \pm 6.38$ & $5.35 \pm 4.42$ \\
& & $43.77 \pm 8.59$ & $29.12 \pm 8.32$ \\
\hline
\end{tabular}

Se presentan las micrografías en la Fig. 2; revelando la morfología aserrada para el acero AISI 4140, para un tiempo de permanencia de $2 \mathrm{~h}$ a una temperatura de 950 (Fig. 2b) y $1000^{\circ} \mathrm{C}$ (Fig. 2d). El tipo de morfología de la capa generada en este trabajo, como las propiedades mecánicas resultantes depende de la composición química, calidad en la pasta de boro, temperaturas de tratamiento y tiempo de borurado. 

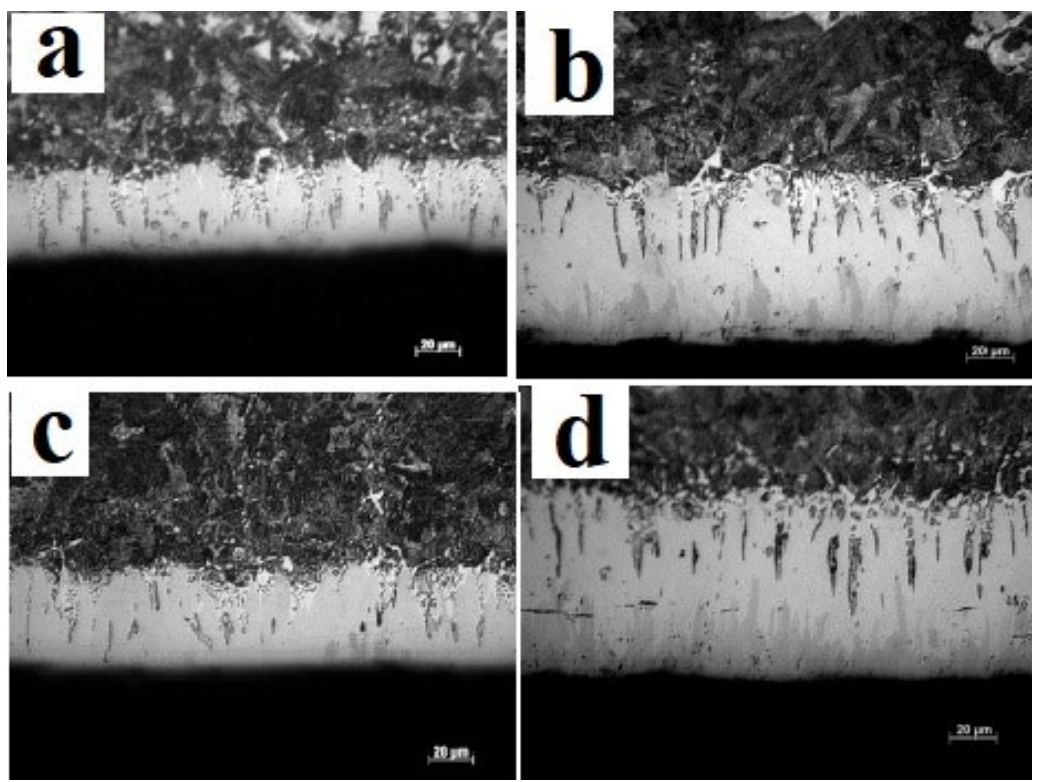

Fig. 2. Perfil del boruro de hierro formado en el acero AISI 4140 a una temperatura $950^{\circ} \mathrm{C}$, tiempo de permanencia; a) $0.5 \mathrm{~h}$ y b) $2 \mathrm{~h}$ y temperatura $1000^{\circ} \mathrm{C}$, con tiempo de permanencia; c) $0.5 \mathrm{~h}$ y d) $2 \mathrm{~h}$.

La Tabla II y III muestran los resultados de dureza y módulo de elasticidad para una carga de $250 \mathrm{mN}$; en función de los resultados experimentales, los resultados de dureza dan a demostrar que los boruros de hierro formados en la superficie del acero AISI 4140 adquieren un factor de dureza mayor al del material base.

TABLA II

Promedio de MiCrodureza del BorURo DE HIERRO EN ACERO AISI 4140 A $950^{\circ} \mathrm{C}$.

\begin{tabular}{cccc}
\hline $\begin{array}{c}\text { Tiempo } \\
\text { (h) }\end{array}$ & Fases & $\begin{array}{c}\text { Módulo de } \\
\text { Elasticidad } \\
\text { (Gpa) }\end{array}$ & $\begin{array}{c}\text { Dureza } \\
\text { (Hv) }\end{array}$ \\
\hline 2.0 & $\mathrm{Fe}_{2} \mathrm{~B}$ & $288 \pm 4.8$ & $1465.42 \pm 66.29$ \\
2.0 & $\mathrm{FeB}$ & $317 \pm 4.3$ & $2132.66 \pm 32.72$ \\
0.5 & $\mathrm{Fe}_{2} \mathrm{~B}$ & $233 \pm 3.9$ & $1083.03 \pm 30.23$ \\
0.5 & $\mathrm{FeB}$ & $277 \pm 3.3$ & $1930.34 \pm 50.06$ \\
\hline
\end{tabular}

TABLA III

Promedio de MiCrodureza del BorURo de HIERRO EN ACERo AISI 4140 A $1000^{\circ} \mathrm{C}$.

\begin{tabular}{cccc}
\hline $\begin{array}{c}\text { Tiempo } \\
\text { (h) }\end{array}$ & Fase & $\begin{array}{c}\text { Módulo de } \\
\text { Elasticidad } \\
\text { (Gpa) }\end{array}$ & $\begin{array}{c}\text { Dureza } \\
\text { (Hv) }\end{array}$ \\
\hline 2.0 & $\mathrm{Fe}_{2} \mathrm{~B}$ & $327 \pm 5.5$ & $2072.62 \pm 22.19$ \\
2.0 & $\mathrm{FeB}$ & $337 \pm 4.5$ & $2141.61 \pm 60.30$ \\
0.5 & $\mathrm{Fe}_{2} \mathrm{~B}$ & $279 \pm 4.3$ & $1375.79 \pm 73.98$ \\
0.5 & $\mathrm{FeB}$ & $281 \pm 3.6$ & $1828.92 \pm 36.81$ \\
\hline
\end{tabular}


Se pudo determinar mediante gráficas esfuerzo-desplazamiento, el comportamiento a la dureza del material, el comportamiento del acero AISI 4140 , con una temperatura a $1000^{\circ} \mathrm{C}$, con un tiempo de exposición de $2 \mathrm{~h}$, presenta una deformación similar al del acero de $950^{\circ} \mathrm{C}$ pero se evidencia un desplazamiento menor, generado por las altas durezas del recubrimiento, se determina su comportamiento por fases obtenidas en la difusión de átomos de boro (véase Fig. 3), se analiza el comportamiento de la penetración después de la indentación, evidenciando que el material base tiene menos dureza, que el material borurado; así mismo, la fase FeB tiene mayor resistencia a la penetración comparado a la fase $\mathrm{Fe}_{2} \mathrm{~B}$.
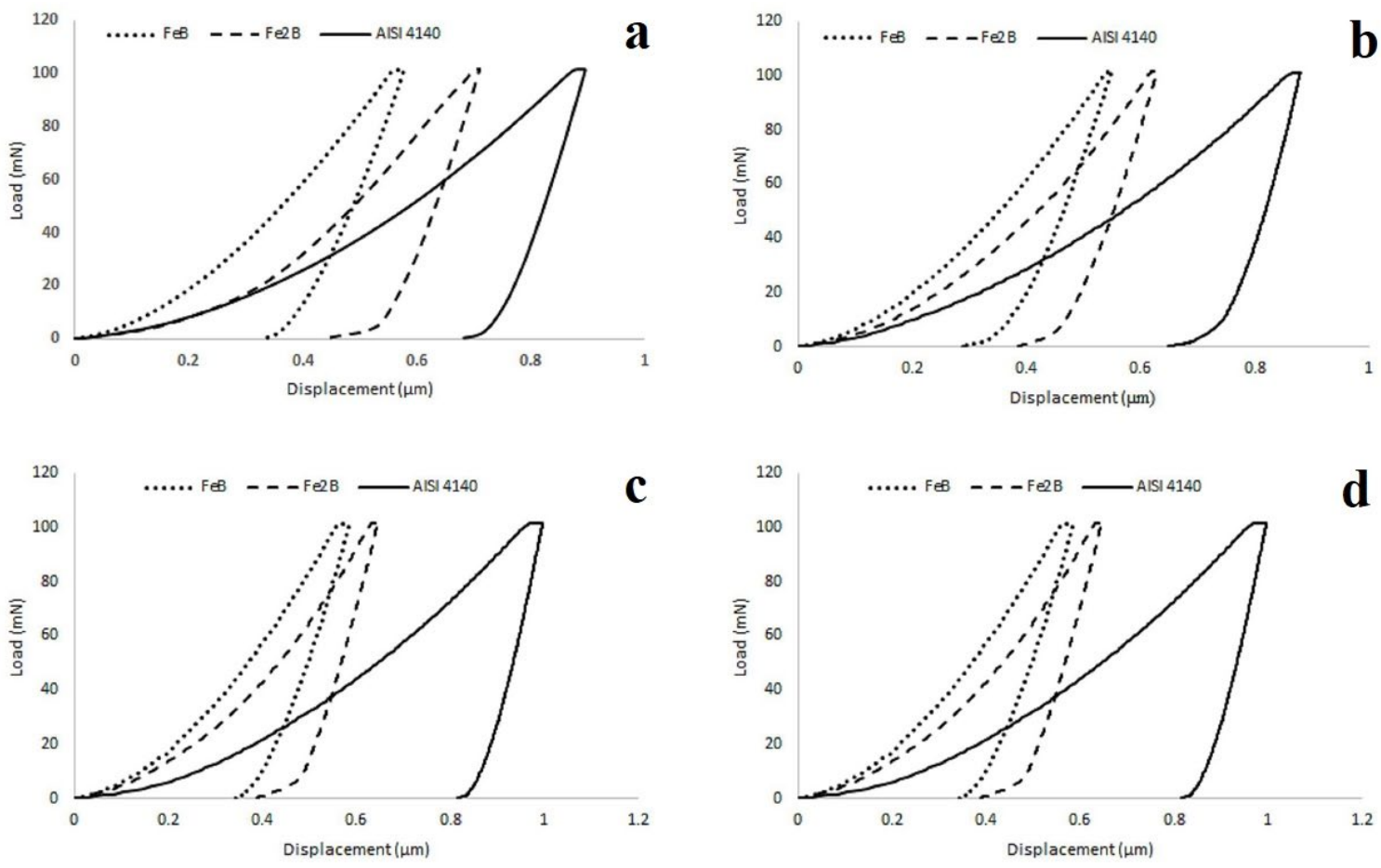

Fig. 3. Gráficas esfuerzo-desplazamiento del comportamiento a la penetración de las fases, en el material AISI 4140 con un tiempo de exposición a) 0.5 y b) $2 \mathrm{~h} \mathrm{a} 950^{\circ} \mathrm{C}$, con un tiempo de exposición c) 0.5 y d) $2 \mathrm{~h} \mathrm{a} 1000^{\circ} \mathrm{C}$.

El patrón de difracción de rayos $\mathrm{X}$ se presenta en la Fig. 4a, muestra la presencia de la fase $\mathrm{Fe}_{2} \mathrm{~B}$ y $\mathrm{FeB}$; además, en la Fig. $5 \mathrm{~b}$ se evidencia con el EDS la presencia de los elementos aleantes sobre la superficie del acero AISI 4140 borurado para las probetas tratadas termoquímicamente a la temperatura de a $1000^{\circ} \mathrm{C}$ con $2 \mathrm{~h}$ de tiempo de exposición. Los XRD (Fig. 4a) y el EDS (Fig. 4b), muestran la formación de la capa bifásica FeB y $\mathrm{Fe}_{2} \mathrm{~B}$ en este estudio. 

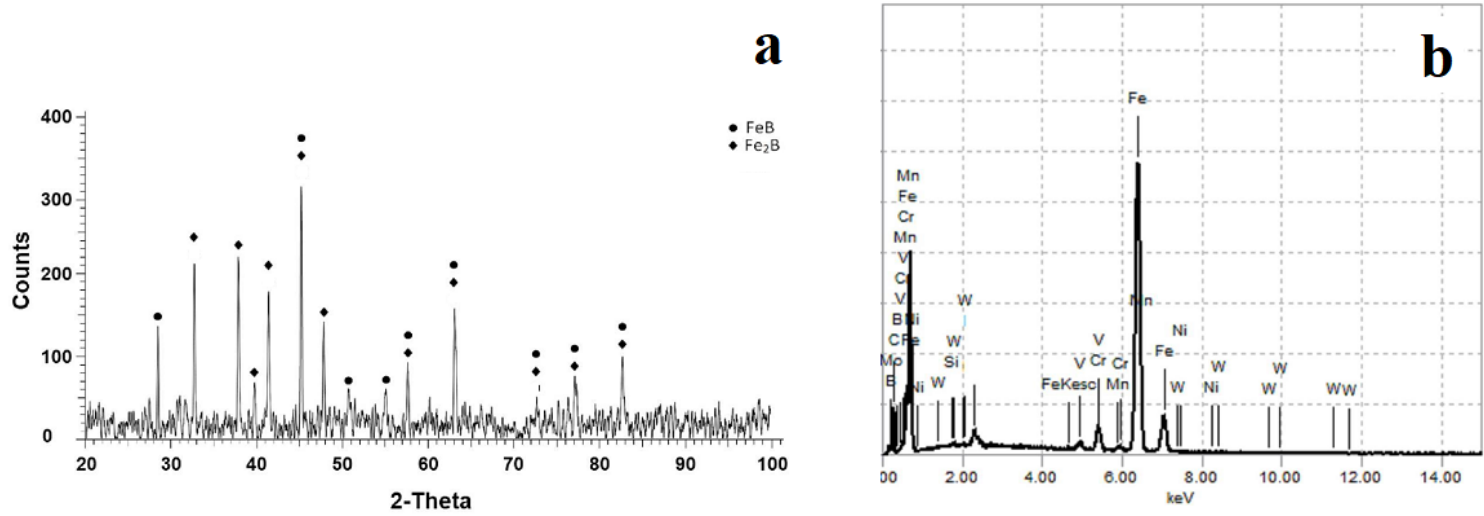

Fig. 4. Patrón de Rayos XRD (a) y EDS (b); sobre el AISI 4140, borurado a temperatura de $1000^{\circ} \mathrm{C}$ con $2 \mathrm{~h}$ de exposición.
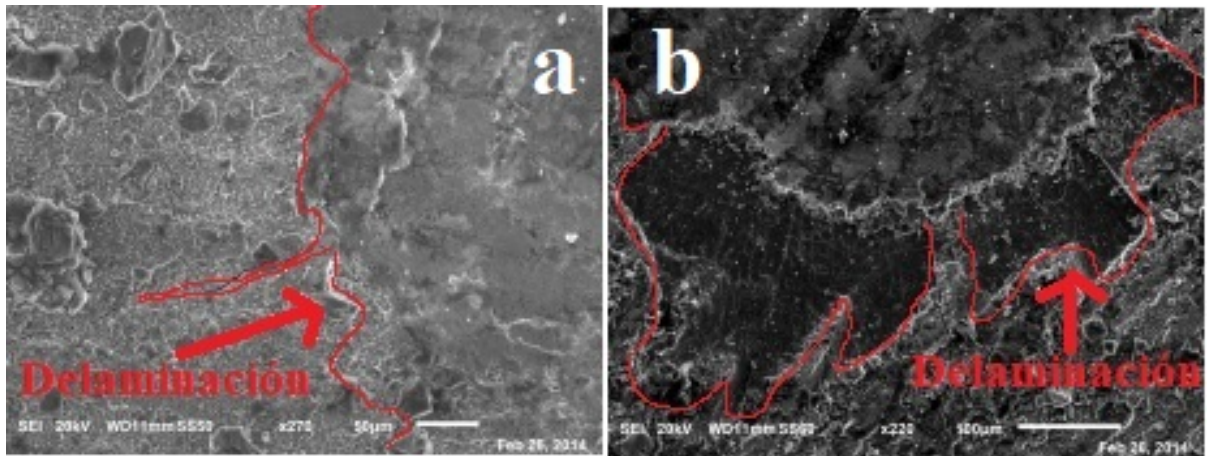

Fig. 5. Delaminación de especímenes borurados a 1273 k con a) 0.5 h y b) 2 h de exposición.

Por otra parte, en este tipo de ensayos se genera una zona central, con fallo cohesivo; se tiene una zona intermedia con insuficiencia cohesiva y adhesiva, causada por esfuerzos generados por la técnica de ensayo Rockwell [8], [9], [10]. En la Fig. 6 se observa delaminación, encontrándose entre rangos de HF3 y HF4, además se observan grietas largas; estos especímenes fueron expuestos a una temperatura de $1000^{\circ} \mathrm{C}$. La Fig. 6a y la Fig. 6b, muestran un incremento en la delaminación del boruro de hierro, además una mayor cantidad de grietas a exposiciones de temperaturas mayores. En la Fig. 6 se observa un comportamiento con una delaminación menor encontrándose entre un rango de HF2 y HF3, estos especímenes fueron expuestos a una temperatura de $900^{\circ} \mathrm{C}$. La Fig. 6a y Fig. $6 \mathrm{~b}$ muestran una delaminación menor en el espécimen evaluado a 0.5 h, observando un comportamiento HF2, teniendo resultados óptimos. Con el espécimen sometido a $2 \mathrm{~h}$, existe delaminación con una adherencia adecuada, teniendo un factor de HF3. Este método cualitativo de adherencia sirve para evaluar la resistencia, rendimiento y falla de los recubrimientos duros a los materiales metálicos, cerámicos, además de los materiales compuestos por mencionar algunos. Se tiene presencia de la fase FeB en estos especímenes; que es propensa a generar grietas por la acción mecánica del ensayo de dureza, debido a tensiones residuales en estos aceros y la alta dureza que tiene esta fase en el recubrimiento 

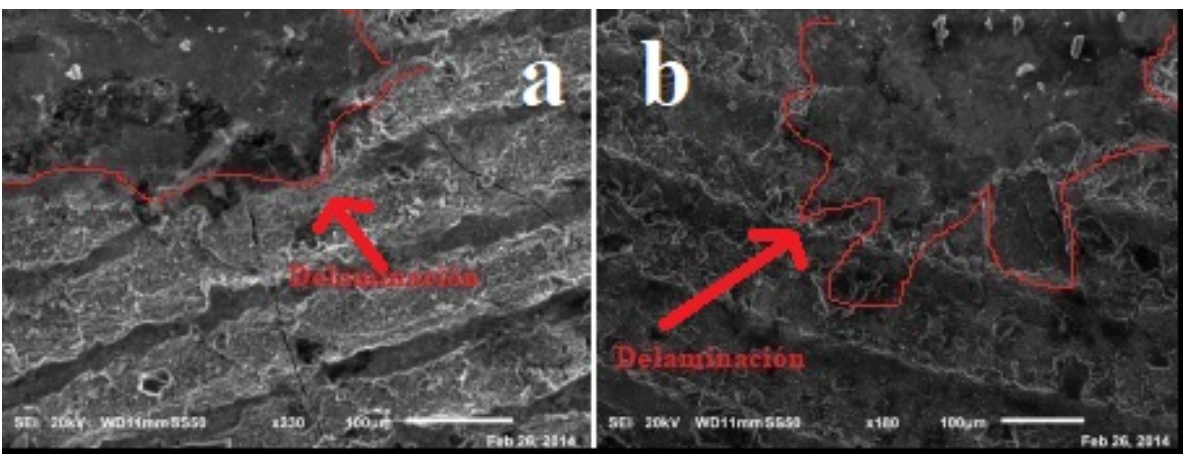

Fig. 6. Delaminación de especímenes borurados a 1223 k con a) 0.5 h y b) 2 h de exposición.

\section{CONCLUSIONES}

Se pudo determinar el crecimiento de la capa de boro, obtenido de las fases $\mathrm{FeB}_{\mathrm{B}} \mathrm{Fe}_{2} \mathrm{~B}$, muestra una difusión con morfología aserrada, aumentando la dureza y la adherencia en el material metálico, los especímenes sometidos a una temperatura de 950 y $1000^{\circ} \mathrm{C}$, con tiempos de exposición de $2 \mathrm{~h}$, tuvieron resultados afines, la mayor difusión observada se presentó en el espécimen sometido a $1000^{\circ} \mathrm{C}$, siendo la fase FeB susceptible a generar mayor cantidad de grietas a menor tiempo de borurización.

La norma VDI, mostro un rango de delaminación de entre HF2 a HF4, la muestra expuesta a una temperatura de $1000^{\circ} \mathrm{C}$ y $900^{\circ} \mathrm{C}$ con una exposición de $2 \mathrm{~h}$, obtuvo una clasificación de HF3 a HF4, con una delaminación mayor, además de un incremento grietas, resulta inadecuada debido a la fragilidad y delaminación mayor que se observa, siendo inconveniente por el desprendimiento de partículas, que para aplicaciones tribológicas resultaría perjudicial expuesta a una temperatura de $1000^{\circ} \mathrm{C}$ y $900^{\circ} \mathrm{C}$ con una exposición de $0.5 \mathrm{~h}$ confirma una aceptable adhesión el recubrimiento duro. Definiendo que, a tiempos menores de $0.5 \mathrm{~h}$, se obtiene una adhesión HF2 óptima del recubrimiento por la poca presencia de la fase FeB.

Se pudo determinar el crecimiento de la capa de boro, obtenido de las fases $\mathrm{FeB}_{ } \mathrm{Fe} \mathrm{e}_{2} \mathrm{~B}$, muestra una difusión con morfología aserrada, aumentando la dureza y la adherencia en el material metálico, los especímenes sometidos a una temperatura de $950^{\circ} \mathrm{C} \mathrm{y} 1000^{\circ} \mathrm{C}$, con tiempos de exposición de $2 \mathrm{~h}$, tuvieron resultados afines, la mayor difusión observada se presentó en el espécimen sometido a $1000^{\circ} \mathrm{C}$, siendo la fase $\mathrm{FeB}$ susceptible a generar mayor cantidad de grietas a menor tiempo de borurización.

La norma VDI, mostro un rango de delaminación de entre HF2 a HF4, la muestra expuesta a una temperatura de $1000^{\circ} \mathrm{C}$ y $900^{\circ} \mathrm{C}$ con una exposición de $2 \mathrm{~h}$, obtuvo una clasificación de HF3 a HF4, con una delaminación mayor, además de un incremento grietas, resulta inadecuada debido a la fragilidad y delaminación mayor que se observa, siendo inconveniente por el desprendimiento de partículas, que para aplicaciones tribológicas resultaría perjudicial expuesta a una temperatura de $1000^{\circ} \mathrm{C} \mathrm{y} 900^{\circ} \mathrm{C}$ con una exposición de $0.5 \mathrm{~h}$ confirma una aceptable adhesión el recubrimiento duro. Definiendo que, a tiempos menores de $0.5 \mathrm{~h}$, se obtiene una adhesión HF2 óptima del recubrimiento por la poca presencia de la fase FeB. 


\section{REFERENCIAS}

[1] W. Heinke, A. Leyland, A. Matthews, G. Berg, C. Friedrich, E. Broszeit, "Evaluation of PVD nitride coatings, using impact, scratch and Rockwell-C adhesion tests," Thin Solid Films, vol. 270, 431-438, 1995.

[2] K. Holmberg, A. Matthews, Properties, “Techniques and Applications in Surface Engineering," Coatings Tribology, Elsevier Science Press, vol. 56, pp. 65-70, 1994, doi: https://doi.org/10.1016/0040-6090(95)06934-8

[3] A. Meneses Amador, I. Campos Silva, J. Martínez Trinidad, S. Panier, U. Figueroa López, A. Torres Hernández, "An expression to determine the Vickers indentation fracture toughness obtained by the finite element method on $\mathrm{Fe}_{2} \mathrm{~B}$ layers," Surface and Coatings Technology, vol. 215, pp. 285-290, 2013, doi: https://doi.org/10.1016/j.surfcoat.2012.06.091

[4] N. López Perrusquia, I. Campos Silva, J. Martínez Trinidad, A. Avilés, E. Alvarez Castañeda, S. Juárez Torres, "Evaluation of brittle layers obtained by boriding on AISI H13 steels" Advanced Materials Research, vol. 65, pp. 47-52, 2009, doi: https://doi.org/10.4028/www.scientific.net/AMR.65.47

[5] N. López Perrusquia, M.A. Doñu Ruiz, V.J. Cortés Suarez, L. D. Rosado Cruz, D. Sánchez Huitron, "Study of Surface Hardening to Injectors of Gasoline," Advanced Materials Research, vol. 538-541, pp. 239-242, 2012, doi: https://doi.org/10.4028/www.scientific.net/AMR.538-541.239

[6] N. López Perrusquia, M. A. Doñu Ruíz, F. Vásquez-Ramírez, O. Trujillo Alfaro, E.Y. Vargas Oliva, "Study of Adhesion in Steels Surface Hardened," Advanced Materials Research, vol. 535-537, pp. 513-516, 2012, doi: https://doi.org/10.4028/www.scientific.net/AMR.535-537.513

[7] Daimler Benz Adhesion Test, Norma No. 3198, Verein Deutscher Ingenieure (VDI), 1992.

[8] S. Taktak, "Some mechanical properties of borided AISI H13 and 304 steels," Materials and Design, vol. 28, pp. 1836-1843, 2007, doi: https://doi.org/10.1016/j.matdes.2006.04.017

[9] D. Sanchez Huerta, T. De la Mora Ramírez, M.A. Doñu Ruiz, N. López Perrusquia, J.V. Cortés Suarez, "Characterization of Adherence Steels Hardened Superficially," MRS Proceedings, vol. 1616, no. S5C, P041, 2013, doi: http://dx.doi.org/10.1557/opl.2014.232

[10] S. Taktak, "Identification of delamination failure of boride layer on common Cr based steels," Journal of Materials Engineering and Performance, vol. 15, no. 5, pp. 570-574, 2006, doi: https://doi.org/10.1361/105994906X124587 